\title{
A Novel Miniaturized Vivaldi Antenna Using Resonant Structure For Ultra-Wideband Applications
}

\author{
Dongdong Geng, Deqiang Yang, Jiafeng Qu, Hua Xiao \\ School of Electronic Engineering, University of Electronic Science and Technology of China \\ Chengdu, Sichuan, China \\ dqyang@uestc.edu.cn
}

Keywords: Vivaldi antenna, resonant structure, Gain, Group delay, UWB.

\begin{abstract}
A Novel Miniaturized Vivaldi Antenna utilizing resonant structure to extend the low-end bandwidth limitation is presented in this paper. The low-end bandwidth limitation of the proposed antenna has extended to $2.45 \mathrm{GHz}$ from the original $3.07 \mathrm{GHz}$. In addition, the gain of the proposed modified antenna has a significantly improvement compared with the conventional Vivaldi antenna at the same size, the directivity has been improved too in the lower frequency. Moreover, the group delay of the antenna shows that the proposed antenna has a good time-domain response.
\end{abstract}

\section{Introduction}

Ultra wideband antennas as an important part of Ultra Wideband system are widely used in the military field, wireless transmission and biological detection, etc. They have been a rapid development in recent years especially. As the kind of end-fire traveling wave antennas. The Vivaldi antenna is one of the best candidates for the Ultra Wideband antennas due to its good directivity, high gain, perfect time domain performance 、 low cross polarization and so on $[1,2,3]$.

The conventional Vivaldi antennas in order to achieve good performance such as high gain, highly directive radiation patterns, wide bandwidth etc. usually have a very large size $[4$, 5]. This is not a conducive to the direction of miniaturization. A Vivaldi antenna presented in [6] using the tapered slot edge with resonant cavity structure to realize miniaturization but its length is also very long which is $0.43 \lambda_{0}\left(\lambda_{0}\right.$ is the wavelength refers to the minimum operating frequency which $\left|S_{11}\right| \leq$ $-10 d B$ ). According to [7], we can miniaturize the size of the Vivaldi antenna by the ways of lowering the minimum operating frequency.

In this paper, a symmetrical pair of resonant branches has been added on a conventional Vivaldi antenna in order to add the resonant points which can reduce the minimum operating frequency. The lowest operating frequency of the $\left|S_{11}\right| \leq$ $-10 \mathrm{~dB}$ bandwidth has reduced by $620 \mathrm{MHz}$ in the case of the size remains unchanged, the relative bandwidth increased by $15.5 \%$. The gain of the proposed antenna has a significant improvement over almost the whole $\left|S_{11}\right| \leq$ $-10 d B$ bandwidth compared with the conventional Vivaldi antenna. In addition, the group delay keeps relatively flat in the whole operating bandwidth means the proposed antenna can be used in the UWB system.

\section{Antenna design}

The design process to extend the low-end bandwidth limitation of the Vivaldi antenna using resonant structure is shown in Fig. 1. All antennas are printed on an FR4 substrate (dielectric constant is 4.4, loss tangent is 0.02) with the thickness of $1 \mathrm{~mm}$. The dimensions of the three antennas are all $30 \mathrm{~mm} \times 36 \mathrm{~mm}$. The detailed design process is as follows: (Unit: $\mathrm{mm}$ ).

Firstly, design a conventional Vivaldi antenna which was used as the reference antenna is shown in Fig. 1(a). The exponential profile curves E1 can be described by Equation (1).

$\mathrm{x}=c_{1} \times \exp \left[r_{e x} \times(y-6)\right]-\left(c_{1}-c_{2}\right) ;(6 \leq y \leq 36)$

$c_{1}, c_{2}$ and $r_{e x}$ are equal to $0.14,0.15$ and 0.172 , respectively.

Secondly, cut off the outer edge region of the reference antenna which was used to add the resonant branches. It is shown in Fig.1 (b). The exponential profile curves E2 can be described by Equation (2).

$\mathrm{x}=c_{3} \times \exp \left[r_{\operatorname{ex1}} \times(y-6)\right]-\left(c_{3}-c_{4}\right) ;(8.5 \leq y \leq 24)$

$c_{3}, c_{4}$ and $r_{e x 1}$ are equal to $0.5,2.8$ and 0.22 , respectively.

Thirdly, a pair of symmetrical resonant branches structure is inserted on the antenna which was used to add the lower frequency resonant points thus can reduce the size of the antenna. It is shown in Fig. 1(c). The exponential curves E3 and E4 can be described by Equations (3) and (4). 
$\mathrm{x}=c_{5} \times \exp \left[r_{e x 2} \times(y-6)\right]-\left(c_{5}-c_{6}\right) ;(8.5 \leq y \leq 19)$

$\mathrm{x}=c_{7} \times \exp \left[r_{e x 3} \times(y-6)\right]-\left(c_{7}-c_{8}\right) ;(8.5 \leq y \leq 24)$

$c_{5}, c_{6}$ and $r_{e x 3}$ are equal to $1.2,6.5$ and 0.22 , respectively, $c_{7}, c_{8}$ and $r_{e x 4}$ are equal to $2.5,11$ and 0.24 , respectively. Photographs of the fabricated antenna are shown in Fig. 2. A tapered micro-strip feeding line was adopted to achieve prefect impedance matching. The width of the port is $2 \mathrm{~mm}$ which can get $50 \Omega$ characteristic impedance. All parameters were shown in Table 1.

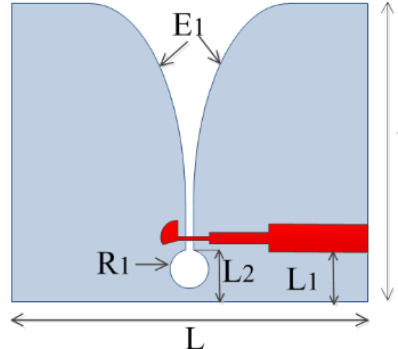

(a)
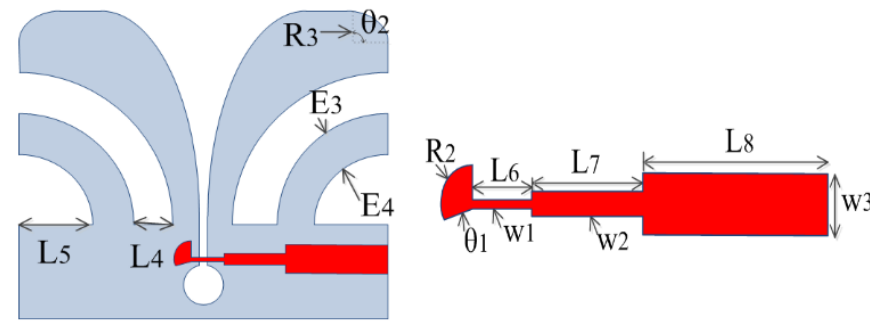

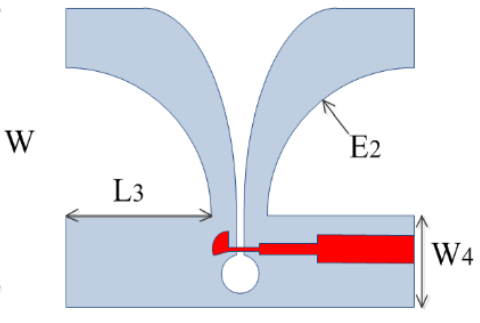

(b) (c)

Fig.1. Configurations of the three Vivaldi antennas: (a) reference antenna (b) Type-A antenna (c) Type-B antenna

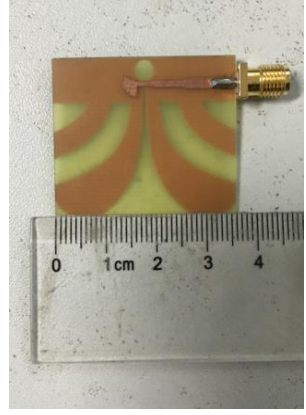

(a)

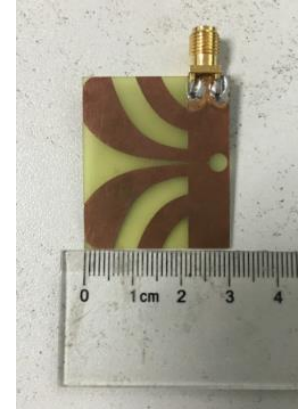

(b)
Fig.2. Photographs of the fabricated antenna (a) Top view. (b) Bottom view

\begin{tabular}{|c|c|c|c|}
\hline parameter & value & parameter & value \\
\hline$L$ & $36 \mathrm{~mm}$ & $W$ & $30 \mathrm{~mm}$ \\
\hline$L_{1}$ & $4 \mathrm{~mm}$ & $W_{1}$ & $0.8 \mathrm{~mm}$ \\
\hline$L_{2}$ & $4.2 \mathrm{~mm}$ & $W_{2}$ & $1.3 \mathrm{~mm}$ \\
\hline$L_{3}$ & $15.2 \mathrm{~mm}$ & $W_{3}$ & $2 \mathrm{~mm}$ \\
\hline$L_{4}$ & $3.7 \mathrm{~mm}$ & $R_{1}$ & $1.9 \mathrm{~mm}$ \\
\hline
\end{tabular}

\begin{tabular}{|c|c|c|c|}
\hline$L_{5}$ & $7 \mathrm{~mm}$ & $R_{2}$ & $2.9 \mathrm{~mm}$ \\
\hline$L_{6}$ & $3 \mathrm{~mm}$ & $R_{3}$ & $3.5 \mathrm{~mm}$ \\
\hline$L_{7}$ & $6.2 \mathrm{~mm}$ & $\theta_{1}$ & $115^{\circ}$ \\
\hline$L_{8}$ & $11.3 \mathrm{~mm}$ & $\theta_{2}$ & $90^{\circ}$ \\
\hline
\end{tabular}

TABLE 1: Optimized parameter of the proposed antenna

\section{Results and analysis}

In order to validate whether the design of the proposed antenna can achieve miniaturization, the Type-B antenna has been fabricated and tested. The radiation patterns and gain of the Type-B antenna are measured using the Microwave anechoic chamber, $\left|\mathrm{S}_{11}\right|$ and Group delay are measured using the Network Analyzer N5230A. Simulation using the software High Frequency Structure Simulator (HFSS).

\subsection{Return loss}

The simulation $\left|S_{11}\right|$ of the reference antenna and Type-B antenna are shown in the Fig. 3. The lower-end $\left|S_{11}\right| \leq$ $-10 \mathrm{~dB}$ limitation of the reference Vivaldi antenna is 3.07 $\mathrm{GHz}$, while the Type-B antenna reduces the limitation to 2.45 GHz. The measured $\left|S_{11}\right|$ of the Type-B antenna is also drawn in Fig. 3. From the fig.3. We can see that the measured lowerend $\left|S_{11}\right| \leq-10 \mathrm{~dB}$ limitation is $2.55 \mathrm{GHz}$. The difference between the simulated and measured results of the Type-B antenna and there is a bulge in $9 \mathrm{GHz}$ are probably due to the effect of the SMA connector and the inaccuracy in fabrication.

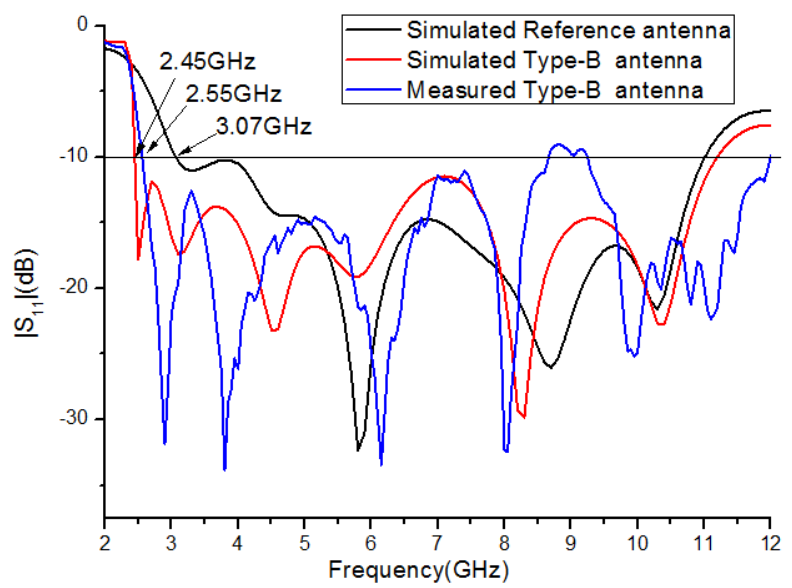

Fig.3. $\left|S_{11}\right|$ of the reference antenna and Type-B antenna 


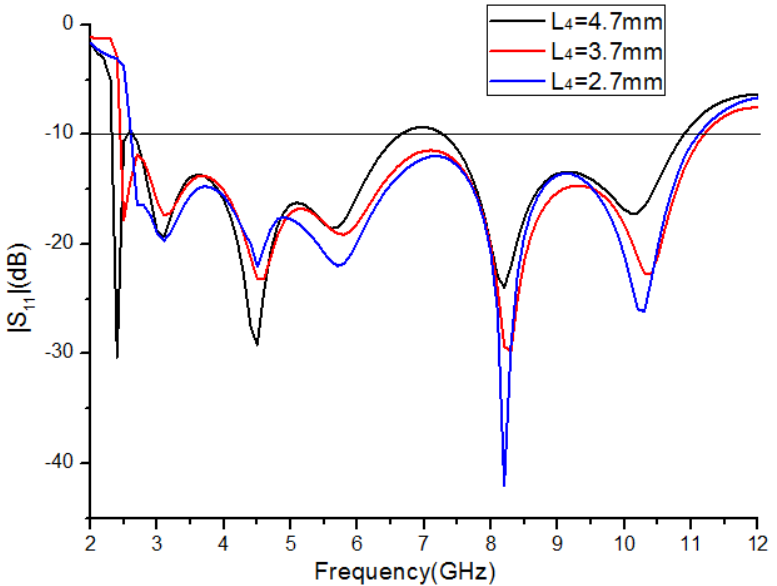

Fig.4. Simulated $\left|S_{11}\right|$ of the Type-B antenna with different value of $L_{4}$.

The effect of paramater $L_{4}$ (major changes the value of $c_{4}$ ) on $\left|S_{11}\right|$ of the proposed antenna is simulated and shown in Fig.4. It is observed that increase the length of the $L_{4}$ can get the lower cutoff frequency. When $L_{4}=4.7 \mathrm{~mm}$ the minimum cutoff frequency is $2.32 \mathrm{GHz}$, but the $\left|S_{11}\right|$ at $2.6 \mathrm{GHz}$ and $7 \mathrm{GHz}$ are above $-10 \mathrm{~dB}$. When $L_{4}=3.7 \mathrm{~mm}$ the propose antenna can get a relatively good return loss, the minimum cutoff frequency is $2.45 \mathrm{GHz}$.

\subsection{Surface current}

In order to further understand the principle of adding the resonant structure can achieve the minimum cutoff frequency. The surface current of the Type-B antenna at 2.45 and $3.1 \mathrm{GHz}$ are shown in Fig. 5. We found that $3.1 \mathrm{GHz}$ is excited by the inside edge of the traditional slot line, while $2.45 \mathrm{GHz}$ is excited by the Semi-enclosed cavity which was composed by E2 and E3. It means that the effective length of the surface current path on the Type-B antenna is lengthened due to the adding resonant structure.

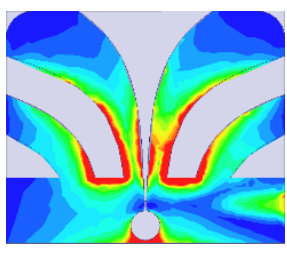

(a)

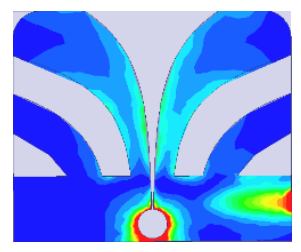

(b)
Fig.5. Surface current distribution of the Type-B antenna at (a) $2.45 \mathrm{GHz}$, (b) $3.1 \mathrm{GHz}$

\subsection{Radiation patterns}

The simulated and measured radiation patterns of the Type-B antenna in E-plane (xoy-plane) and H-plane (yoz-plane) at 2. $45 \mathrm{GHz} 、 4 \mathrm{GHz} 、 7 \mathrm{GHz}$ and $10 \mathrm{GHz}$ are depicted in Fig. 6. In addition, the simulated radiation patterns of the reference antenna is also added in Fig.6. From the Figure we can see that the directivity of the Type-B antenna has a significant improvement compared with the reference antenna in the low frequency. The difference between the simulated and measured results of the Type-B antenna are probably due to the effect of the SMA connector and the inaccuracy in fabrication. (Unit: $\mathrm{dB}$ ).

\subsection{Gain and Group delay}

According to the analysis of surface current distribution, the resonant structure contributes to lengthening the effective length of the surface current path to achieve the lower frequency resonant points. The simulated gain of reference antenna and the simulated and measured gain of Type-B antenna are shown in Fig. 7. From the figure we can see that the gain of the Type-B antenna can achieve a significant increase in the low frequency and intermediate frequency. In addition, the measured result is in great agreement with the simulation, the difference probably due to the effect of the inaccuracy in fabrication.
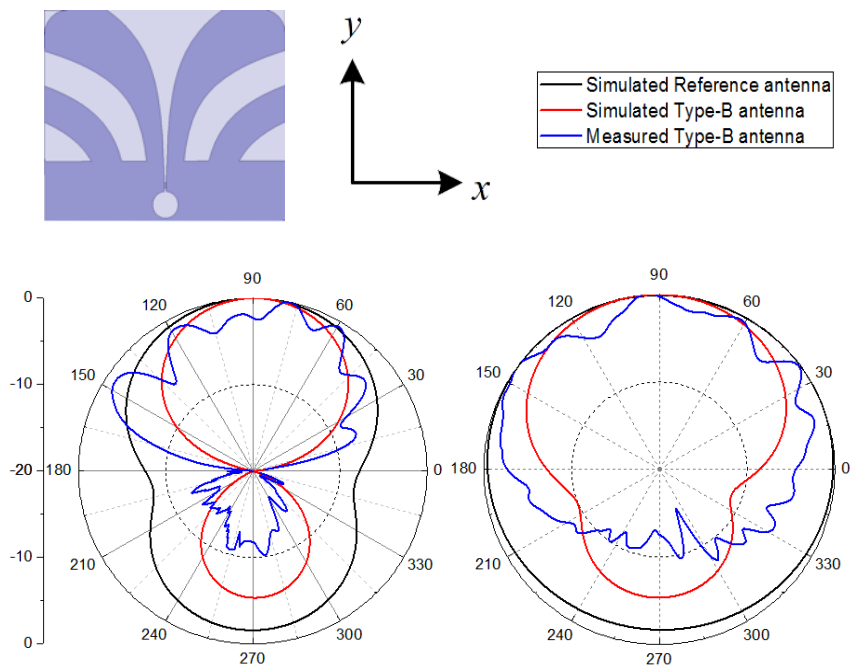

(a)

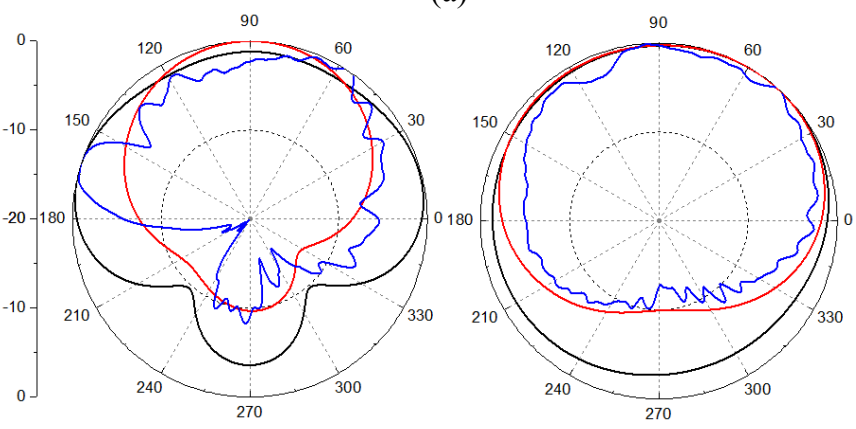

(b) 


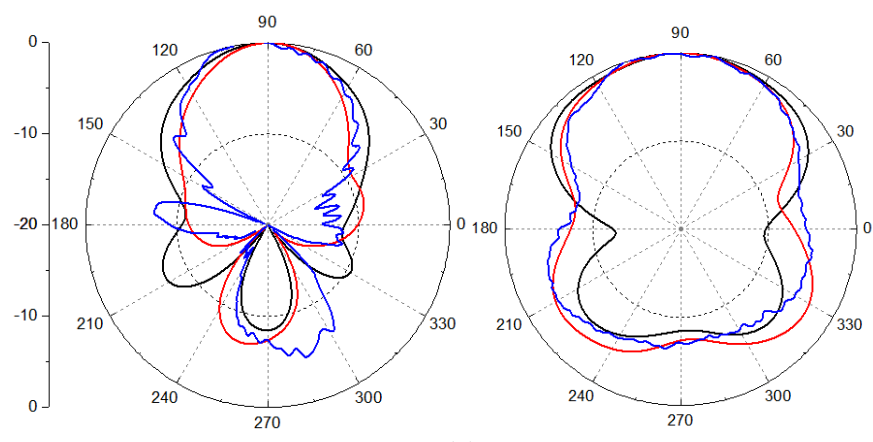

(c)
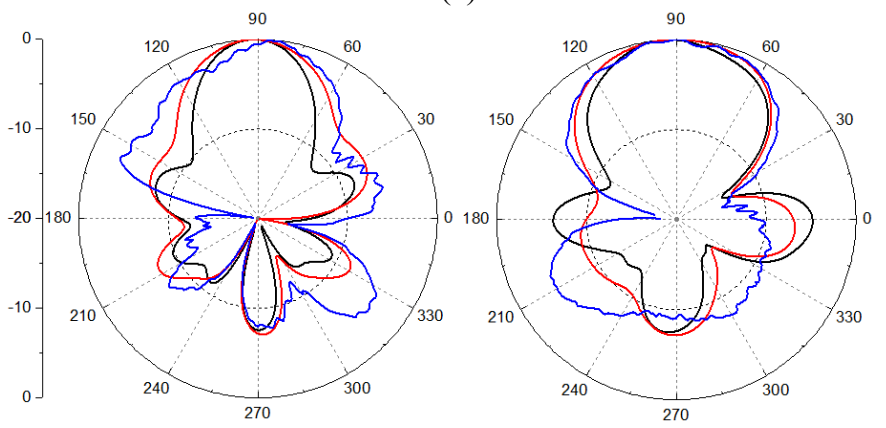

(d)

E-plane (xoy-plane)

H-plane (yoz-plane)

Fig.6. Radiation patterns of the Reference antenna and Type-B antenna in E-plane (xoy) and H-plane (yoz) at (a) $2.5 \mathrm{GHz}$, (b) $4 \mathrm{GHz}$, (c) $7 \mathrm{GHz}$, (d) $10 \mathrm{GHz}$.

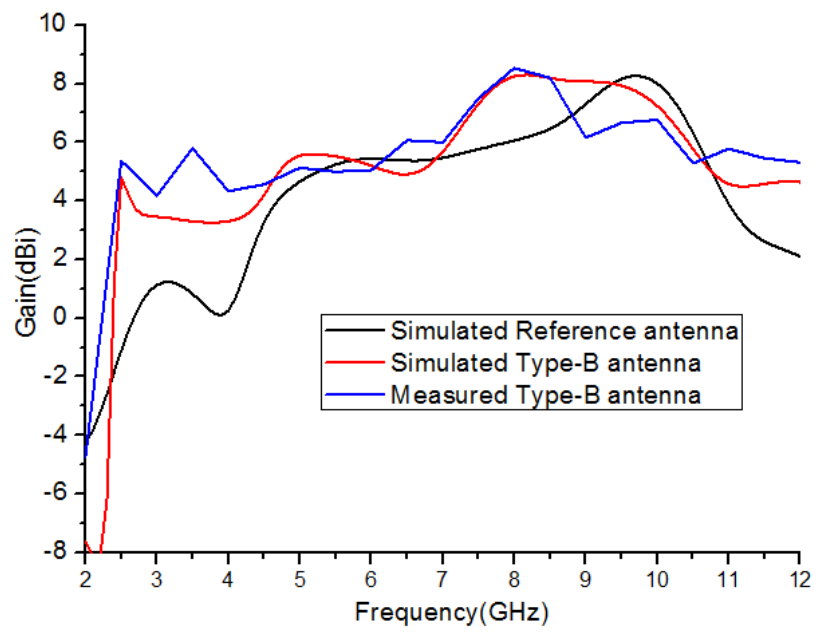

Fig.7. Gain of the proposed Type-B antenna

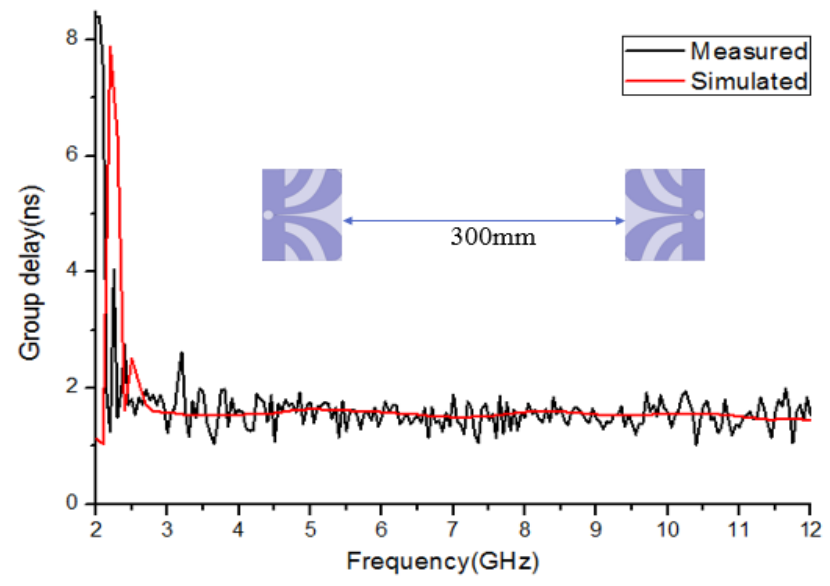

Fig.8. Measured and Simulated group delay of the Type-B antenna

Group delay is an important parameter for an UWB antenna because it can measure the waveform distortion in time domain. Therefore, the group delay of the Type-B antenna is also analysis, two identical antennas are placed face to face in a distance of $300 \mathrm{~mm}$ to analog signal transceiver experiment. The measured and simulated group delay are shown in Fig. 8. From the figure we can see that the measured group delay of the Type-B antenna is around $1.6 \mathrm{~ns}$ in the entire operating frequency bandwidth with a variation less than $0.4 \mathrm{~ns}$. It is a relatively flat group delay response.

\section{Conclusions}

In this paper, a planar printed Vivaldi antenna with resonance structure is designed and experimentally studied for UWB applications. The lower-end $\left|S_{11}\right| \leq-10 \mathrm{~dB}$ limitation of the proposed antenna has extended to $2.45 \mathrm{GHz}$ from the original $3.07 \mathrm{GHz}$, the size of the Vivaldi antenna reduced from $0.31 \lambda_{0} \times 0.37 \lambda_{0}$ to $0.25 \lambda_{0} \times 0.30 \lambda_{0}$, which is a quit compact size for the Vivaldi antennas. In addition, the gain and radiation direction are improved at low frequencies by using the resonance structure. Moreover, the measured group delay of the Type-B antenna shows that the proposed antenna has a good time-domain response in the whole operating bandwidth.

\section{References}

[1] G.R. Aiello and G.D. Rogerson, "Ultra-wideband wireless systems", IEEE Microwave Mag. volume. pp. 36-47, (2003).

[2] S. H. He, "An improved Vivaldi antenna for vehicular wireless communication systems", IEEE Antennas Wireless Propag. Lett. volume. pp. 1505 -1508, (2014).

[3] E. W. Reid , L. Ortiz-Balbuena , “A. Ghadiri and K. Moez, "A 324-element Vivaldi antenna array for radio astronomy instrumentation", IEEE Trans.Antennas Propag. volume. pp. 241 -249, (2012).

[4] X. L .Kang, Z .R .Li, "An original Vivaldi antenna for 1$8 \mathrm{GHz}$ wideband application", 2015 IEEE $6^{\text {th }}$ International 
Symposium on Microwave. Antenna Propag, pp. 231-233, (2015).

[5] P. Fei, Y. C. Jiao, W. Hu and F. S. Zhang, "A miniaturized antipodal Vivaldi antenna with improved radiation characteristics", IEEE Antennas Wireless Propag. Letter., volume. pp. 127-130, (2011).

[6] Y. S. Liu, W.J. Zhou, "A Novel Miniaturized Vivaldi Antenna Using Tapered Slot Edge with Resonant Cavity
Structure for Ultra-wide Band Applications", IEEE Antennas and Wireless Propag. Letter. volume. pp. 18811884, (2015).

[7] K. Ebnabbasi, D. Busuioc, R. Birken and M. Wang, "Taper design of Vivaldi and co-planar Tapered Slot Antenna (TSA) by Chebyshev transformer", IEEE Trans. Antennas Propag. volume. pp. 2252-2259, (2012). 